\title{
Rapid field identification of subjects involved in firearm-related crimes based on electroanalysis coupled with advanced chemometric data treatment
}

Xavier Cetó, ${ }^{1,2}$ Aoife M. O’ Mahony, ${ }^{1}$ Izabela A. Samek, ${ }^{1}$ Joshua R. Windmiller, ${ }^{1}$ Manel del Valle, ${ }^{2}$ Joseph Wang ${ }^{1}$

${ }^{1}$ Department of Nanoengineering, University of California, San Diego, La Jolla, CA 92093, USA. tel.: +1 (858) 246 0128; fax: +1 (858) 534 9553; e-mail: josephwang@ucsd.edu

${ }^{2}$ Sensors \& Biosensors Group, Department of Chemistry, Universitat Autònoma de Barcelona, Edifici Cn, 08193 Bellaterra, Spain. tel.: +34 93 5811017; fax: +34 93 5812379; e-mail: $\underline{\text { manel.delvalle@uab.es }}$

\begin{abstract}
We demonstrate a novel system for the detection and discrimination of varying levels of exposure to gunshot residue from subjects in various control scenarios. Our aim is to address the key challenge of minimizing the false positive identification of individuals suspected of discharging a firearm. The chemometric treatment of voltammetric data from different controls using Canonical Variate Analysis (CVA) provides several distinct clusters for each scenario examined. Multiple samples were taken from subjects in controlled tests such as secondary contact with gunshot residue (GSR), loading a firearm and post-discharge of a firearm. These controls were examined at both bare carbon and gold-modified screen-printed electrodes using different sampling methods: the 'swipe' method with integrated sampling and electroanalysis, and a more traditional acid-assisted q-tip swabbing method. The electroanalytical fingerprint of each sample was examined using square-wave voltammetry; the resulting data were processed with Fast Fourier Transform (FFT), followed by CVA treatment. High levels of discrimination were thus achieved in each case over 3 classes of samples (reflecting different levels of involvement), achieving maximum accuracy, sensitivity and specificity values of $100 \%$ employing the leave-one-out validation method. Further validation with the 'jack-knife' technique was performed and the resulting values were in good agreement with the former method. Additionally, samples from subjects in daily contact with relevant metallic constituents were analyzed to assess possible false positives. This system may serve as a potential method for a portable, field-deployable system aimed at rapidly identifying a subject who has loaded or discharged a firearm to verify involvement in a crime, hence providing law enforcement personnel with an invaluable forensic tool in the field.
\end{abstract}

Keywords: gunshot residue, canonical variate analysis, square-wave voltammetry, identify shooter.

\section{Introduction}


The rapid detection of gunshot residues from an individual suspected of discharging or handling a firearm can provide valuable information in a multitude of scenarios in forensic investigations. ${ }^{1-4}$ Data from the Criminal Justice Statistics Center of the California Department of Justice show that over a 10 year period (1994-2004), firearms were the most commonly used weapon in homicide cases, constituting $72.6 \%$ of the weapons used. This level of firearm-related crime necessitates more forensic analysis of physical evidence in conjunction with police investigations. The subject of forensic analysis has thus received enormous recent attention. Extensive literature is available regarding detection of both organic and inorganic constituents of gunshot residue (GSR) using a variety of analytical techniques. ${ }^{5-8}$ Most of the methods used are bulky, expensive and require trained personnel operating within the confines of centralized laboratory facilities. ${ }^{9-11}$ The lack of sufficient specificity for the determination of whether or not a suspect has discharged a firearm or was merely in the proximity of such an event must also be addressed.

We demonstrate here, for the first time, a potential method for the effective discrimination of different control experiments in the determination of levels of contact with GSR from the hands of different subjects using the integration of electrochemical signals with advanced chemometric data treatment. Our goal has been to examine and maximize variations in the signals generated by multiple organic and inorganic GSR constituents from subjects who have discharged a firearm compared with those who have had secondary contact with GSR (in the presence of other shooters or contact with GSRcontaminated surfaces).

The coupling of advanced multi-variate calibration techniques and chemometric tools with electroanalysis represents an alternative to classic chromatographic and spectrometric methods for the simultaneous determination of compounds, synthesizing the entire recorded electrochemical signal to maximize the information content. ${ }^{12}$ The most common multi-variate methods employed for qualitative analysis are Principal Component Analysis (PCA) and Linear Discriminant Analysis (LDA). These methods employ different mathematical transformations applied to the sample responses (either the raw recorded signal or the extracted coefficients after preprocessing) to build a model that allows more straightforward visualization of the similarities and differences between the different analyzed samples. ${ }^{13,14}$ These principles can be brought to a higher complexity level if an array of voltammetric sensors with a complementary response is used in what is known as an electronic tongue analysis system. ${ }^{15}$ Chemometric data treatment has received growing attention towards the analysis of GSR in recent years. One such complementary approach is the integration of Raman spectroscopy and PCA, which has been successfully demonstrated to differentiate GSR produced by the discharge of two different ammunition-firearm combinations. ${ }^{16}$ The sequence of voltammetry and PCA has been demonstrated recently as a method to discriminate the type of firearm used by a suspect. ${ }^{17}$ However, there are no reports aimed at addressing the key forensic challenge of discriminating between subjects who have discharged a firearm and those who have not under a variety of different control scenarios (including those with secondary contact with GSR).

The method described herein couples field-deployable electrochemical stripping measurements with the Canonical Variate Analysis (CVA) data processing/clustering strategy. We examined cyclic squarewave voltammetry of samples taken from subjects in different control scenarios over a wide cathodic 
and anodic potential window, covering the redox processes of multiple organic and inorganic constituents of GSR. Previous attempts at electrochemical detection of GSR using anodic stripping voltammetry (ASV) for residue detection in conjunction with swabbing or tape-lift sampling methods have proved successful for detecting inorganic species such as $\mathrm{Sb}, \mathrm{Pb}$ and $\mathrm{Cu}$ at both hanging drop mercury electrodes ${ }^{1,9}$ and mercury-film glassy carbon electrodes. ${ }^{10,11}$ Unfortunately, mercury electrodes cannot be used for routine decentralized forensic analysis. Recently, the simultaneous electrochemical detection of both metallic and organic species consistent with GSR has been demonstrated by our group at a carbon electrode in a single voltammetric run, to further enhance the information content and reliability of forensic GSR investigations. ${ }^{8}$

The present work combines such information-enhanced electrochemical analysis with powerful CVA data processing with the goal of obtaining effective and rapid discrimination between subjects who have discharged a firearm and those who have not under a variety of relevant control scenarios. CVA analysis was thus used to classify samples from each control set, from two different points of view. This outlines a distinction between those who have had no contact with GSR, those who have had contact, albeit of the secondary form, and those who have directly handled a firearm, regardless of discharge within this study. Consistent CVA discrimination - with well-defined minimally-dispersed clusters - is thus illustrated for several different control signals taken before and after the discharge of the firearm for each subject examined herein. Such distinction may hold considerable importance in future applications to discriminate between direct involvement in a firearm-related crime or the mere presence in the vicinity of the discharge of a firearm.

To implement such voltammetric/CVA discrimination, we relied on two different sampling methods to obtain the gunshot residue for analysis. One of the sample collection methods employed in this work is a recently developed simple and fast integrated sampling/measurement 'swipe' method. ${ }^{18}$ The use of disposable carbon sensor strips in this method ensures that a low cost and reliable electrochemical fingerprint is obtained from the firearm discharge samples compared to expensive, cumbersome laboratory equipment. We also utilized a traditional GSR sampling method involving dilute acid-assisted q-tip swabbing for comparison. ${ }^{10,11}$

The voltammetric/CVA analysis described here may be incorporated into a hand-held device to allow portability and speed in future forensic applications. This could potentially serve as a system to provide a simple response outlining a subject's complicity in a crime involving the discharge of a firearm, whereby categories including 'free-to-go', 'witness to a shooting' and 'involved in a shooting' can be ascertained from this methodology.

\section{Experimental}

\subsection{Chemicals and materials}

Acetate buffer ( $\mathrm{pH}$ 4.6) was purchased from Fluka (St. Louis, MO). Gold plating solution ( $\mathrm{KAuCN}_{2}$, ORO Temp24 RTU RACK) was purchased from Technic Inc. (Anaheim, CA). Deionized water (18 $\mathrm{M} \Omega-\mathrm{cm}$ ) from a NANOpure Diamond system (Thermo Scientific, Dubuque, IA) was used to prepare all solutions. 


\subsection{Instruments and procedure}

Electrochemical measurements were performed using an $\mu$ Autolab II (Eco Chemie, The Netherlands). Screen-printed carbon electrodes (SPCEs) were used for all measurements. The carbon ink used for the working and counter electrodes was Acheson Electrodag 440B (Henkel Electronic Materials LLC). The fabrication of these SPCEs has been detailed in the literature. ${ }^{19}$ Au-modified SPCEs were modified using a gold plating solution $\left(\mathrm{KAuCN}_{2}\right)$ in accordance to a recent report. ${ }^{18}$

\subsection{Square-Wave Voltammetry}

Cyclic square Wave Voltammetry (SWV) was employed to characterize GSR electrochemical signatures. ${ }^{8}$ At bare carbon SPEs, oxidative SWV signals were obtained with an initial potential of -1.3 $\mathrm{V}$ vs. $\mathrm{Ag} / \mathrm{AgCl}$, maintained for $120 \mathrm{~s}$, and subsequently scanned to a final potential of $+1.3 \mathrm{~V}$ vs. $\mathrm{Ag} / \mathrm{AgCl}$. Reductive signals were implemented from initial potential $1.3 \mathrm{~V}$ (vs. Ag/AgCl), maintained for $120 \mathrm{~s}$, and subsequently scanned to a final potential $-1.3 \mathrm{~V}$. At Au-modified electrodes, oxidative SWV signals were obtained with an initial potential of $-0.8 \mathrm{~V}$ vs. $\mathrm{Ag} / \mathrm{AgCl}$, maintained for $120 \mathrm{~s}$, and subsequently scanned to a final potential of $+0.3 \mathrm{~V}$ vs. Ag/AgCl. All scans were performed at a frequency of $25 \mathrm{~Hz}$, amplitude of $25 \mathrm{mV}$ and potential step of $4 \mathrm{mV}$. All experiments were conducted in acetate buffer (pH 4.6).

\subsection{Sampling of Gunshot Residue}

Integrated sampling of GSR directly from the hand of a shooter was performed at a local shooting range (P2K, El Cajon, CA).

Swiping samples were isolated by abrasively rubbing the electrode surface over the hand of the suspect. The sensor strip electrode was held at the silver electrical contacts by the sampler. Two types of swiping samples were taken: (a) the 3-electrode strip surface was swept over the back of the subjects firing hand 7 times; (b) from the thumb and back of the firing hand 5 times each. Samples from the back of the hand were taken by swiping the 3-electrode surface over the back of the hand of the subject exactly 7 times in a downward motion from wrist to knuckle. For sample collection from the thumb and back of the hand, the 3-electrode surface was swiped over the back of the hand 5 times in a downward motion from wrist to knuckle, and over the thumb of the subject 5 times in a downward motion from tip to joint. These guidelines ensure that multiple operators can conduct this sampling while maintaining consistency. Each electrode was then placed in an individual resealable storage bag to prevent crosscontamination. For analysis, a $50 \mu \mathrm{L}$ aliquot of acetate buffer was dropped onto the electrode surface (upon which the GSR sample has already been immobilized), and SWV was carried out as per Section 2.3.

Swabbing samples were performed using a sample collection kit consisting of plastic handled cotton tipped swabs, $2 \mathrm{~mL}$ glass sample vials and a $5 \%(\mathrm{v} / \mathrm{v}) \mathrm{HNO}_{3}$ solution in a squeeze bottle. The GSR sample was collected by soaking the cotton tipped swab with 3-5 drops of the $\mathrm{HNO}_{3}$ solution and swabbing the back of the hand. The cotton tip was then cut from the stem with scissors and placed in the glass vial. $1.00 \mathrm{~mL}$ of $4 \mathrm{M} \mathrm{HCl} / 0.1 \mathrm{M}$ acetate buffer was added to each sample. The samples were allowed to soak overnight (12-16 h) before analysis. For analysis, a $40 \mu \mathrm{L}$ aliquot of acetate buffer was 
dispensed onto the electrode surface. The surface was then spiked with $10 \mu \mathrm{L}$ of the GSR sample from the $4 \mathrm{M} \mathrm{HCl} / 0.1 \mathrm{M}$ acetate buffer mixture containing the cotton swab, and SWV was performed, as per Section 2.3.

Sampling was not duplicated for any subject. Rather, comparison between samples from 6 different subjects per control scenario was deemed sufficient to demonstrate precision for these experiments.

For bare carbon SPEs, samples were obtained at different instances during the experimental process: in the laboratory (prior to any contact with GSR), named $\mathrm{N}$-No contact; in the lobby of the shooting range, (without entering the lanes where others were discharging firearms), named S-Secondary contact; at the shooting lanes where others were discharging firearms (without handling or discharge), named $P$ Presence at discharge; having handled and loaded the firearm (without discharge), named L-Load; after firing several rounds from the weapon (10 rounds from a Glock 9mm or 8 rounds for a Sig Sauer 45) F-Fire; after washing the hands (with soap and water) - W: Wash. 6 different subjects with 6 control scenarios resulted in 36 samples in total. We obtained these samples utilizing the swiping protocol (36 samples), and the swabbing protocol (35 samples due to an error in one of the samples).

For Au-modified SPEs, samples were obtained for 4 different subjects with 5 control scenarios $(N$ No contact, P-presence at discharge, L-Load, F-Fire and W-Wash) utilizing the swiping protocol of sample collection. This resulted in a total of 20 samples. The voltammetry for this work has been previously reported, ${ }^{18}$ and since this was conducted prior to the experiments at the bare carbon SPE, fewer control scenarios and fewer subjects were involved. Subsequent to this data set, the method was enhanced with an extra control scenario (S-Secondary contact) and the number of subjects was increased to facilitate a more robust CVA model.

\subsection{Firearms and Ammunition}

The firearms used in this work were a Glock 17 9mm and a Sig Sauer P220 45 caliber. The leaded ammunition used for the Glock was Remington $\mathrm{UMC}^{\circledR}$ Target 9mm luger and the leadless ammunition was Remington $\mathrm{UMC}^{\circledR} 9 \mathrm{~mm}$ luger. The leaded ammunition used for the Sig Sauer was Remington $\mathrm{UMC}^{\circledR}$ Target 45 automatic.

\subsection{Data processing}

Prior to building the classification model, a preprocessing step for data reduction was performed on the square-wave voltammograms obtained at the sensor strip electrodes, employing Fast Fourier Transform (FFT). ${ }^{20}$ In this manner, both the cathodic and the anodic waves of the SWV were compressed separately and a number of obtained coefficients were used as inputs in the classification model.

Classification of samples was achieved by means of CVA analysis, which used a stepwise inclusion

method to allow the removal of low-contributing variables to the classification model. ${ }^{21}$ Moreover, given that this is a supervised method, classification success was evaluated using a 'leave one out' crossvalidation scheme. Additionally, to demonstrate, the efficacy of the proposed method, model classification success was further validated utilizing the 'jack-knife' method $^{22}$ which, in turn, enabled the estimation of the associated standard errors. 
Chemometric processing of the data was performed by specific routines in MATLAB 7.1 (MathWorks, Natick, MA) written by the authors.

\section{Results and Discussion}

This work focuses on the detection of GSR, obtained from the hand of a subject, over various control scenarios. The following sections demonstrate the unique classification protocol among subjects involved in the handling or discharge of a firearm compared to subjects who have been in contact with gunshot residue from a secondary source. Voltammetric analysis was performed employing two types of sensor strip electrode and from samples collected using two separate methods (outlined in Section 2.4). This was followed by chemometric CVA treatment of the electrochemical data. Samples of GSR from different control scenarios were acquired as per Section 2.4 and voltammetry was carried out as per section 2.3. The voltammetric data were then preprocessed according to Section 3.1 below. This rapid and effective identification of subjects who have discharged a firearm, along with the control tests, make the development of this new electrochemical/CVA method promising for potential use in forensic investigations of firearm-related crimes.

\subsection{FFT signal preprocessing}

To fully exploit all the information obtained from each voltammogram and to prevent the saturation of the associated classification model with excessively complex data, a compression step was performed to decrease the dimensionality of the electrochemical signatures. In addition, this step may also help to avoid redundancy in the input data and to obtain a more robust classification model with better generalization ability since high-frequency 'noise' is eliminated while preserving the 'signal' envelope. ${ }^{23}$

This compression step was achieved by means of a Fast Fourier Transform (FFT), which is particularly useful because of its ability to compress data and remove noise at the same time. In this way, compromising between the reconstruction degree and the number of obtained coefficients, raw voltammetric data was compressed up to only 64 coefficients without any loss of significant information, which allowed a compression of the original information up to $88.0 \%$ (71.7\% in the case of Au-modified electrodes).

Although good representation of the original data could be achieved with fewer coefficients, thus increasing compression ratio, it was preferred to ensure the best reconstruction degree given CVA was performed using a stepwise inclusion method which allows for the removal of the variables that have a lower contribution to the classification model. That is, having a list of independent variables, some of which may be useful predictors, but some of which are almost certainly useless, the aim is to find the best subset to carry out the task of prediction as well as possible, with as few variables as possible. Hence, this method is very effective in selecting and removing the variables that do not contribute at all to the prediction success.

\subsection{Classification models}


CVA was used to classify samples from two different points of view for each set of samples. In the first case, discrimination between the six types of samples outlined in Section 2.4 (N-No contact, SSecondary contact, P-Presence at discharge, L-Load, F-Fire and W-Wash) was attempted; in a second approach, discrimination of the samples was simplified to a 3-class study case: Free $(N)$, Witness ( $S \&$ $P$ ) and Involved ( $L, F \& W$ ), outlining a subjects complicity in a firearm-related crime in a simpler manner. Although this entails a loss of information, it may improve the reliability of the method. This outlines a distinction between those who have had no contact with GSR, those who have had contact, albeit secondary, and those who have directly handled the firearm, regardless of discharge. In particular, the control scenarios S-Secondary contact and P-Presence were, in part, chosen to reflect a subject in daily contact with GSR-constituent materials. Such distinction may hold much importance demonstrating implication in a crime as well as minimizing false positive identifications.

After pre-processing the recorded voltammograms with FFT, the obtained coefficients were used as input into the CVA model, which was employed to execute the classification of the samples. Given that this is a supervised method, classification success was evaluated using leave-one-out cross validation. In this manner, each sample is classified by means of the analysis function derived from the other samples (all cases except the case itself). This process was repeated $k$ times (as many as samples) leaving out one different sample each time, the one to be classified, which acts as model validation sample. Thus, with this approach all samples are used once as validation.

Upon completion of the CVA modelling, the 'jack-knife' method was used to evaluate the performance of the model. ${ }^{22,24}$ The jack-knife method was originally suggested in statistical analysis as a general approach for testing hypotheses and calculating confidence intervals in situations where apparently no better methods could be used. With this approach, the samples are first split into training and testing subsets, then a model is constructed with data from the training subset and its performance is evaluated using testing subset. Standard errors are calculated from different data subdivisions of training and test subsets with random distribution and repeating the modelling stage. In this manner, it may be implemented via either excluding one sample, or even several samples, during each iteration. ${ }^{24}$ This method has the advantage that it avoids dependence on predictions from the specific subdivision of data, thus providing a more realistic evaluation of the proposed approach given that, in each case, a new model is trained and evaluated with a new external test subset of samples which are not employed in any way in the modelling routine. The 'goodness' of fit is, accordingly, a valid measure of the modelling performance. In this way, train/test data subdivision is repeated randomly $k$ times, evaluating the model's response for the test validation subset during each iteration and using unbiased data.

In our case, the 'jack-knife' method was used training with $80 \%$ of the data and tested with the remaining $20 \%$. This subdivision of the original data set was subsequently repeated 10 times, excluding different test samples during each iteration. Each excluded sample was selected randomly to ensure the veracity of the model as well as to guarantee that performance does not depend on the specific subsets used. Finally, model performance was evaluated from the mean of the replica results, which, in turn, allows for the calculation of the precision and confidence intervals for the obtained results.

\subsubsection{SPCE swipe}


A bare SPCE was used to measure the organic and inorganic species present in GSR over a wide potential window. The 'swiping' sampling technique, outlined in Section 2.4, has been described in previous work. ${ }^{18}$ We examined samples taken from both the thumb and back of the hand, as well as the back of the hand only. A clearer discrimination profile and thus more favorable results were obtained from samples taken from the thumb and back of the hand, and these are displayed below. However, it should be noted that clear discrimination from the samples taken from the back of the hand only were also achieved, with sensitivity and specificity values noted below. We examined both the oxidation and reduction profiles of the GSR samples. The advantage of this method is to extract additional information of the anodic and cathodic signatures of other GSR components such as organic compounds and other metals at more negative potentials, which would lead to a richer departure point, improving the model response and resulting in a less expensive sensor. In the course of this work, we analyzed the voltammetry from six subjects for the six different control scenarios outlined in Section 2.4 (resulting in 36 samples in total in each study).

Figure 1A displays an example of some of the voltammetric signals resulting from the samples taken from subjects at the gun range using the firearms and ammunition described in Section 2.5. The voltammetry from samples for L-Load, F-Fire \& W-Wash are shown. Acetate buffer was dispensed onto the electrode surface subsequent to sampling and SWV was carried out in the potential range of $-1.3 \mathrm{~V}$ to $+1.3 \mathrm{~V}$ vs. Ag/AgCl (outlined in Section 2.3). The oxidative voltammetry exhibits 3 stripping signals at potentials $-0.8 \mathrm{~V},-0.275 \mathrm{~V}$ and $0 \mathrm{~V}$ vs. Ag/AgCl. These are attributed to anodic stripping of metals, possibly zinc + nickel amalgams, lead and copper, respectively. The signals for lead and copper ${ }^{8}$ and for nickel-zinc alloys ${ }^{25}$ have already been observed at similar potentials at a glassy carbon electrode (GCE). Each of these species is noted to be present in the ammunition according to the material safety data sheets. The signal at $0 \mathrm{~V}$ (vs. Ag/AgCl) is much greater for the L-Load scan than it is for any of the other scans. This signal is attributed to $\mathrm{Cu}$ and the increase in the signal for the $L$-scan may be attributed to increased contact of $\mathrm{Cu}$ from the brass bullet case. This electrochemical behavior has previously been observed by the authors using the 'swipe' method of GSR collection. ${ }^{18}$ Further signals are observed at more positive potentials also, which may be attributed to the organic components of GSR. Anodic signals are observed at $0.25 \mathrm{~V}, 0.6 \mathrm{~V}$, and $1.098 \mathrm{~V}$ (vs. Ag/AgCl). These may be attributed to oxidation of diphenyl benzene (DPB), diphenylamine (DPA) and nitroglycerin (NG), respectively. Both DPA and NG are noted to be present in all the ammunition according to the material safety data sheet and DPB is formed upon the oxidation of DPA. ${ }^{26}$ The signals for these species have previously been observed at similar potentials at a GCE during investigations of the metallic and organic constituents of GSR. ${ }^{8}$ Upon the cathodic sweep of the GSR samples, a signal is observed in the F-Firing scan at a potential of 0.625 vs. Ag/AgCl, which may be attributed to the reduction of DPA. This voltammetric fingerprint has again been examined previously by the authors at a GCE. ${ }^{8}$ The use of this 'cyclic' SWV waveform to deliver such a detailed electroanalytical fingerprint, in a single voltammetric run, demonstrates the intrinsic advantages of simplicity and rapidity of this method for the detection of the components of GSR.

Clear discrimination of the samples was achieved at these electrodes upon CVA treatment of this data, as seen in Figure 1B, with patterns in the figure evidencing that samples are grouped according to the exposition level, where these well established clusters clearly separate the 6 classes of samples. 
Classification results of the CVA leave-one-out cross-validation approach are summarized in Table 1. As expected from the CVA plot, nearly all samples were correctly classified according to exposure level. Hence, the classification rate of the samples reached an accuracy of $86.1 \%$. The efficiency of the classification obtained was also evaluated according to its sensitivity, i.e. the percentage of objects of each class identified by the classifier model, and to its specificity, the percentage of objects from different classes correctly rejected by the classifier model. The value of sensitivity, averaged for the classes considered was $86.1 \%$, and that of specificity was $97.2 \%$. This method provides clear discrimination of various control scenarios for a number of subjects over a very short timescale with facile sampling and analysis. As mentioned above, CVA was also performed on samples taken using the swiping method from the back of the hand only and resulted in a classification rate $66.7 \%$, sensitivity of $66.7 \%$ and specificity of $93.3 \%$.

To improve model reliability and to provide a simpler response outlining a subject's complicity, classification of samples was also attempted shrinking previous data to a 3-class study case. As per Figure 1B, a new CVA model was built and evaluated using the leave-one-out cross validation method. In this case, only three groups were considered, Free, Witness and Involved, and the CVA model was formed by just two canonical variables (CVs). The same data used for Figure 1B was also used in this model, whereby Free encompasses $\mathrm{N}$-No contact, Witness uses the previous data for S-Secondary contact and P-Presence at discharge, and Involved uses the data for L-Load, F-Fire and W-Wash.

Figure 1C outlines the clear discrimination for the samples based on the three exposure level categories. Patterns in the figure evidence that samples are grouped according to degree of involvement. According to this classification, the Free cluster is still far removed from the other two clusters, which have similar merits for CV 1, but that are clearly separated by CV 2. Similar behavior is observed for Witness and Involved, whereby no overlap with the other categories was observed and clear discrimination along the axes was obtained, providing a simple system for the discrimination of subjects in different scenarios based on their exposure to GSR. Furthermore, as with the previous case for 6 categories (Figure 1B), a confusion matrix was constructed employing a leave-one-out cross-validation approach, the results of which are summarized in Table 2. As expected from the CVA plot, all samples were correctly classified, with a classification rate of $100 \%$ in terms of accuracy. Also the same values for sensitivity and specificity were obtained for the method. An interesting noticeable feature is the reduced dispersion on the attained grouping, especially in contrast with the high separation between groups; this feature may constitute highly reliable decision systems, where 'no doubt' cases appear.

\subsubsection{SPCE swab}

Swabbing assisted with diluted acid is traditionally used to obtain GSR samples from a subject suspected of discharging a firearm for electroanalysis. ${ }^{10,11}$ As per Section 3.2.1, a new CVA model was constructed to assess the discrimination capabilities when swabbing was utilized as the method to obtain GSR samples from a suspect. These samples were also measured at bare SPCEs. The sampling method is outlined in Section 2.4, and the samples taken for different control scenarios are the same as those outlined in Section 3.2.1 (resulting in 35 samples; due to error 1 sample was lost). 
Figure 2A shows the score plot of the first two canonical variables obtained after CVA analysis of GSR samples according to 6 classes (observed in Figure 1B), while Figure 2B displays the classification of swabbing samples according to a simpler response mode (observed in Figure 1C). We observe larger dispersion along cluster centroids in both Figures 2A and 2B, compared to the 'swiping' procedure of Figure 1, although discrimination for all the classes is still achieved.

Examining the obtained scores plot in Figure 2A, we observe that $N$-No contact samples appear further from the other classes, but not by as much as in previous cases using the 'swiping' method of sample collection. That is, $N$-No contact samples have lower CV 2 score values; $P$-Presence and $S$ Secondary contact samples have similar intermediate CV 2 score values, being discriminated by CV 1; and W-Wash, F-Fire and L-Load samples present the same behavior. An analogous model was obtained for the simpler approach (previously observed in Figure 1C), but with clearly less dispersion along the centroids; i.e. groups sorted according to exposure level along CV 2, Free group further from the others, and Involved and Witness groups mainly separated by CV 1.

A confusion matrix was built employing a leave-one-out cross-validation approach for both cases, with results provided in Table 1 (Figure 2A) and Table 2 (Figure 2B). For the 6 classes study case (Figure 2A, Table 1), as expected from the CVA plot, predictive performance was lower than that achieved for the 'swiping' method of sample collection, with a classification rate of $77.1 \%$ in terms of accuracy. Also, the model's sensitivity and specificity were demoted, with values of $77.2 \%$ and $95.5 \%$, respectively. However, in the case of the 3-class study shown in Figure 2B (Table 2), better results were obtained with a classification rate of $91.4 \%$, sensitivity of $90.5 \%$ and $95.8 \%$ for specificity. This classification method may prove more useful for future field-deployable applications for identification of involvement in a crime as it displays higher values of sensitivity and specificity regardless of the sampling method. Despite this however, it is clear that the 'swiping' method of sample collection provides higher discrimination than that of the 'swabbing' method. This is highly advantageous as the former is cheaper and faster than the latter, and more suited to in-field sampling and analysis by minimally-trained operators.

\subsubsection{AuSPCE swipe}

Finally, results employing bare SPCE electrodes were compared with those previously obtained employing gold-modified electrodes, the voltammetry of which has already been reported. ${ }^{18}$ In this case, samples were taken from 4 subjects in the following scenarios (details of which are outlined in Section 2.4): N-No Contact; P-Presence at discharge; L-Load; F-Fire; W-Wash (resulting in 20 samples in total). Based on the results previously gathered and following the same procedure for data treatment, new models were built and the results obtained are presented in Figure 3.

Figure 3A displays analysis from the 5 different scenarios mentioned above and demonstrates clear discrimination obtained between the different types of samples, with well-established clusters for each of the expected classes and low dispersion along each class centroid. Analyzing the obtained plot, it can be interpreted that sample clusters are sorted along CV 1 based on the exposure level to GSR. That is, as we move along the ' $\mathrm{CV} 1$ ' axis from left to right, we see a trend based on the exposure to GSR from $N$ No Contact to F-Fire, relating position/score with the exposition level. Meanwhile 'CV 2' mostly 
discriminates load and fire clusters from the rest. It is interesting to note that Wash samples have intermediate score values between classes with less exposure ( $N$-No Contact or P-Presence at discharge) and those with higher exposure (L-Load and F-Fire), which may be expected from deliberate removal of a significant degree of GSR from the hands of the subjects. Even under such scenarios, it is still possible to distinguish the cases with use of the model. The confusion matrix was built and predictive evaluator's indexes were extracted (Table 1). In this case, the classification rate reached $80.0 \%$, sensitivity was $80.0 \%$ and specificity was $95.0 \%$.

To improve the model's response and increase classification rate, a new model based on three classes similar to those observed in Figure 1C and 2B, was built: Free $(N)$, Witness $(P)$ \& Involved $(L, F$ $\& W$ ). Figure $3 \mathrm{~B}$ outlines the clear discrimination for the samples based on the three exposure level categories. Patterns in the figure evidence that samples are grouped according to degree of involvement. Moreover, samples could be distinguished by only the scores of CV 1, where CV 2 mainly separates Free from Witness and Involved clusters, which share more similarities. In this way, using the new samples division (Table 2), the classification rate improved to $100 \%$, with the same values obtained for the method's sensitivity and specificity. As previously noted in Section 3.2.2, this classification method appears to be more advantageous in the field for identification of involvement in a crime as it displays higher values of sensitivity and specificity regardless of either the electrode surface used or the sampling method implemented. This particular classification system will also allow for a simpler, more robust portable electronic system whereby facile responses to only 3 classes can be obtained, rather than the 6 classes outlined. Future efforts with this approach may involve the simplification of the Free and Witness categories into one to ensure that those in daily contact with GSR-constituent species are not implicated. Further refinement can then be executed on the Involved category to examine the level of involvement.

\subsubsection{Minimization of false positive identification of a shooter}

To further test and validate the system in the interest of preventing false positive identification occurrences, we examined the voltammetric signature and resulting chemometric data generated from samples taken from machine-shop technicians and smokers. The machine-shop technicians were selected as they would be in contact with metals consistent with GSR through daily activities such as operating heavy machinery, painting, milling, and welding. Smokers were selected to observe whether there would be any similarities between the organic species found in cigarette smoke and gunshot residue. The samples were obtained from the thumb and back of the hand of the subject using the swiping method, as per Section 2.4. The sampling protocol followed was identical to the one employed to build the model, illustrated in Fig. 1B. The samples were examined using square-wave voltammetry as per Section 2.3 and the data were processed and input into the previously-implemented model reported in Figure 1B; thus, those samples did not contribute to the training process and were considered as blind samples for the model. The subsequent output is shown in Figure 4, the technicians represented in purple, and the smokers in grey. Of the 12 samples taken (7 technicians, 5 smokers), 1 was classified in S-Secondary contact, 8 in P-Presence at discharge, and 3 in L-Load. While ideally all these samples would have been classified in $N$-No contact, it is not surprising that they are more consistent with 
samples taken after secondary contact from GSR-contaminated surfaces and GSR immobilized by other shooters. It is noteworthy that none of the subjects examined were classified as F-Fire. Future work on the voltammetric/chemometric approach will involve the inclusion of these scenarios and other possible circumstances into the experimental design and modeling steps. The classification may also be simplified using fewer clusters, and further validation can be performed with repetition of these subjects.

\subsection{5 'Jack-knife' validation}

Although results obtained with the leave-one-out strategy sometimes are criticized as overoptimistic, its use is necessary when there is no availability of large sample sets. ${ }^{27}$ In this respect, a second classification model was used to process all of the data reported herein to demonstrate the efficacy of the proposed method. This was achieved using the jack-knife technique, which, in turn, facilitated the calculation of standard errors of the performance indicators. ${ }^{22}$ This method is described in detail in Secion 3.2.

With this approach, the samples are first split into training and testing subsets, then a model is constructed with data from the training subset and its performance is evaluated using testing subset. Thus, in this manner, data used to evaluate the model's performance is not used in its construction, hence facilitating the collection of unbiased and more realistic performance indicators. This separation was performed at random, and the classification model was executed for 10 iterations using different samples for training and validation at each step to ensure that no bias was present. The results of the mean values for accuracy, sensitivity and specificity for 10 different models, for each data set reported are presented in Table 3. When comparing these values to those obtained using the leave-one-out approach, similar behavior is obtained, although results are slightly demoted given the variation in the train/test data proportion (fewer samples are used to build the model, which can decrease its generalization ability). However, the use of this approach also allows the estimation of tolerance values for accuracy, sensitivity and specificity percentages, providing additional information of the precision of the approach.

Nevertheless, it is clear that the variations in the different control scenarios are sufficient to provide acceptable classification between a subject who has discharged a firearm and one who has not. In particular, the values obtained for accuracy, sensitivity and specificity for the preferred method of swiping at a bare carbon SPE using a 3-class system (Free, Witness, Involved) are 96\%, 96\% and 98\%, respectively. This shows the robust nature of of the GSR sensor strip, integration of the sampling protocol with the sensor strip towards efficient collection and successful data reduction and feature extraction. This second prediction set validates the EC/CVA relationship proposed herein for effective discrimination of GSR samples from relevant scenarios.

\section{Conclusions}

We have demonstrated, for the first time, the coupling of advanced electrochemical analysis with powerful multi-variate CVA as a potential method for the rapid identification of a subject who has discharged a firearm utilizing a sensor strip electrode and integrating sampling and analysis. Effective 
discrimination of the level of contact with GSR for different subjects examined in this study has been achieved using a variety of control experiments relevant to various forensic scenarios, as indicated from the corresponding well-defined, minimally-dispersed clusters. Specifically, we examined the voltammetry of samples taken from subjects in several control tests: no exposure to GSR, secondary exposure from surfaces and air, and exposure from loading and firing. The resulting voltammetric/CVA analysis provides distinguishable clusters for each scenario examined, the results of which were validated using two different approaches: leave-one-out and jack-knife technique. The integration of the sampling and analysis through the 'swiping' method of sample collection provides a higher level of discrimination for the different scenarios compared to the more traditional sampling method of

swabbing, ${ }^{9}$ allowing a potentially more rapid and facile system for operatives in the field. The use of a bare carbon sensor strip for this analysis also provides an inexpensive and portable electrode surface. Samples from subjects in occupations with daily exposure to materials consistent with GSR were also examined using the generated CVA model. None of these samples were classified as having fired a weapon, which may minimize false positive identification. While this promising proof-of-concept study demonstrates a novel and potentially attractive system for utilization in criminal investigations, further work is required in order to fully assess the scope of its applications in relevant forensic scenarios. Indeed, a larger pool of samples can improve the robustness of the model; simplification of the controls involving no contact with the firearm will improve the applicability of this system in real-world cases. The specificity, rapidity and portability of this approach indicates promise for a field-deployable, handheld device for investigating firearm-related crimes that may potentially be able to address the key challenge of decreasing the occurrence of false positive identifications for the discharge of a firearm.

\section{References}

1. Briner, R. C.; Chouchoiy, S.; Webster, R. W.; Popham, R. E. Anal. Chim. Acta 1985, 172, 3137.

2. Dahl, D. B.; Cayton, J. C.; Lott, P. F. Microchem. J. 1987, 35, 360-364.

3. Saverio Romolo, F.; Margot, P. Forensic Sci. Int. 2001, 119, 195-211.

4. Brandone, A.; De Ferrari, F.; Pelizza, P.; Signori, M. Forensic Sci. Int. 1990, 47, 289-295.

5. Dalby, O.; Butler, D.; Birkett, J. W. J. Forensic Sci. 2010, 55, 924-943.

6. Laza, D.; Nys, B.; Kinder, J. D.; Kirsch-De Mesmaeker, A.; Moucheron, C. J. Forensic Sci 2007, 52, 842-850.

7. MacCrehan, W. A.; Bedner, M. Forensic Sci. Int. 2006, 163, 119-124.

8. Vuki, M.; Shiu, K.-K.; Galik, M.; O'Mahony, A. M.; Wang, J. Analyst 2012, 137, 3265-3270.

9. De Donato, A.; Gutz, I. G. R. Electroanalysis 2005, 17, 105-112.

10. Woolever, C. A.; Dewald, H. D. Forensic Sci. Int. 2001, 117, 185-190.

11. Woolever, C. A.; Starkey, D. E.; Dewald, H. D. Forensic Sci. Int. 1999, 102, 45-50.

12. Gutiérrez, J. M.; Moreno-Barón, L.; Céspedes, F.; Muñoz, R.; del Valle, M. Electroanalysis 2009, 21, 445-451. 
13. Jolliffe, I. T. Principal Component Analysis; Springer: New York, 2002; p 488.

14. Duda, R. O.; Hart, P. E.; Stork, D. G. Pattern classification; John Wiley \& Sons: New York, 2000; Vol. 2, p 654.

15. del Valle, M. Electroanalysis 2010, 22, 1539-1555.

16. Bueno, J.; Sikirzhytski, V.; Lednev, I. K.. Anal. Chem. 2012, 84, 4334-4339.

17. Salles, M. O.; Bertotti, M.; Paixão, T. R. L. C. Sens. Actuators, B 2012, 166-167, 848-852.

18. O’ Mahony, A. M.; Windmiller, J. R.; Samek, I. A.; Bandodkar, A.; Wang, J. Electrochem. Comm. 2012, 23, 52-55.

19. Windmiller, J. R.; Santhosh, P.; Katz, E.; Wang, J. Sens. Actuators, B 2011, 155, 206-213.

20. Cetó, X.; Céspedes, F.; del Valle, M. Talanta, 2012, 99, 544-551.

21. Johnson, R. A.; Wichein, D. W. Applied multivariate statistical analysis; Prentice-Hall: Upper Saddle River, N.J., 2007; Vol. 6th, p 773.

22. Martens, H.; Martens, M. Food Qual. Prefer. 2000 11, 5-16

23. Despagne, F.; Luc Massart, D. Analyst 1998, 123, 157R-178R.

24. Shao, J.; Tu, D. The Jackknife and bootstrap. Springer: New York, 1995; p 516

25. Elkhatabi, F.; Sarret, M.; Miiller C. J. Electroanal. Chem. 1996, 404, 45-53.

26. Yang, H.; Bard, A.J. J. Electroanal. Chem., 1991, 306, 87-109.

27. Kirsanov, D.; Mednova, O.; Vietoris, V.; Kilmartin, P. A.; Legin, A. Talanta 2012, 90. 109-116

\section{Acknowledgements}

This is supported by the Department of Defense Biometrics and Forensics Office under Contract No. HQ0034-11-C-0034. X. Cetó thanks the support of Dept. d'Innovació, Universitats i Empresa de la Generalitat de Catalunya (Catalonia) for the predoctoral and exchange grants. Manel del Valle receives support from the program ICREA Academia. Any opinions, findings and conclusions or recommendations expressed in this material are those of the author(s) and do not necessarily reflect the views of the Washington Headquarters Services-Acquisition Directorate [Assistant Secretary of Defense for Research and Engineering (Defense Biometrics and Forensics Office)]. We would like to thank that staff at the P2K gun range (El Cajon, CA) for their cooperation. 
Table 1. Confusion matrix built according to gunshot reside (GSR) exposure level obtained using the Canonical Variate Analysis (CVA) model and leave-one-out cross validation for the three set of samples: ${ }^{a}$ swipe screen-printed carbon electrode (SPCE), ${ }^{b}$ swab SPCEand ${ }^{c}$ gold-modified SPCE.

\begin{tabular}{|c|c|c|c|c|c|c|c|c|c|c|c|c|c|c|c|c|c|c|}
\hline$\overline{\text { Found }}$ & \multicolumn{3}{|c|}{$\bar{N}$} & \multicolumn{3}{|c|}{$\bar{S}$} & \multicolumn{3}{|c|}{$\bar{P}$} & \multicolumn{3}{|c|}{$\overline{\mathrm{L}}$} & \multicolumn{3}{|c|}{$\bar{F}$} & \multicolumn{3}{|c|}{$\bar{W}$} \\
\hline Expected & $\mathrm{a}$ & b & $\mathrm{C}$ & $\mathrm{a}$ & b & C & $\mathrm{a}$ & b & c & $\mathrm{a}$ & B & C & $\mathrm{a}$ & b & c & a & b & C \\
\hline$\overline{\mathrm{N}}$ & 6 & 6 & 6 & 0 & 0 & 0 & 0 & 0 & - & 0 & 0 & 0 & 0 & 0 & 0 & 0 & 0 & 0 \\
\hline S & 0 & 0 & 0 & 5 & 5 & 2 & 0 & 0 & - & 0 & 1 & 0 & 0 & 0 & 0 & 1 & 0 & 0 \\
\hline$P$ & 0 & 0 & 0 & 0 & 0 & - & 6 & 4 & - & 0 & 1 & - & 0 & 1 & - & 0 & 0 & - \\
\hline $\mathrm{L}$ & 0 & 0 & 0 & 0 & 0 & 0 & 2 & 1 & - & 4 & 4 & 4 & 0 & 0 & 0 & 0 & 0 & 0 \\
\hline$F$ & 0 & 0 & 0 & 0 & 1 & 0 & 0 & 0 & - & 0 & 1 & 0 & 6 & 2 & 4 & 0 & 2 & 0 \\
\hline W & 0 & 0 & 0 & 0 & 0 & 2 & 0 & 0 & - & 1 & 0 & 0 & 1 & 0 & 0 & 4 & 6 & 2 \\
\hline
\end{tabular}

Table 2. Confusion matrix built according to the 3-class response mode obtained using CVA model and leave-one-out cross validation for the three set of samples: ${ }^{\mathrm{a}}$ swipe SPCE, ${ }^{\mathrm{b}}$ swab SPCE and ${ }^{\mathrm{c}}$ gold modified electrodes.

\begin{tabular}{c|ccc|ccc|ccc}
\hline Found & \multicolumn{3}{|c|}{ Free } & \multicolumn{3}{|c|}{ Witness } & \multicolumn{3}{|c}{ Involved } \\
Expected & a & b & c & a & b & C & a & b & c \\
\hline Free & 6 & 5 & 4 & 0 & 0 & 0 & 0 & 1 & 0 \\
Witness & 0 & 0 & 0 & 12 & 12 & 4 & 0 & 0 & 0 \\
Involved & 0 & 2 & 0 & 0 & 0 & 0 & 18 & 15 & 12 \\
\hline
\end{tabular}

Table 3. Summary of models performance for the different approaches employing both leave-one-out and jack-knife for the validation. In the latter, expressed results correspond to the mean and standard deviation of 10 replicas.

\begin{tabular}{|c|c|c|c|c|c|c|}
\hline & \multicolumn{3}{|c|}{ Leave-one-out } & \multicolumn{3}{|c|}{ Jack-knife } \\
\hline & \%Class & Sensitivity & Specificity & \%Class & Sensitivity & Specificity \\
\hline $\begin{array}{c}\text { CSPE/Swipe } \\
6 \text { classes }\end{array}$ & $86.1 \%$ & $86.1 \%$ & $97.2 \%$ & $85.7 \pm 9.5 \%$ & $85.0 \pm 10.2 \%$ & $97.2 \pm 1.9 \%$ \\
\hline $\begin{array}{l}\text { CSPE/Swipe } \\
3 \text { classes }\end{array}$ & $100 \%$ & $100 \%$ & $100 \%$ & $95.7 \pm 9.6 \%$ & $95.7 \pm 9.6 \%$ & $95.7 \pm 9.6 \%$ \\
\hline $\begin{array}{c}\text { CSPE/Swab } \\
6 \text { classes }\end{array}$ & $77.1 \%$ & $77.2 \%$ & $95.5 \%$ & $74.3 \pm 13.1 \%$ & $74.3 \pm 13.1 \%$ & $74.3 \pm 13.1 \%$ \\
\hline $\begin{array}{l}\text { CSPE/Swab } \\
3 \text { classes }\end{array}$ & $91.4 \%$ & $90.5 \%$ & $95.8 \%$ & $90.0 \pm 9.6 \%$ & $90.0 \pm 9.6 \%$ & $90.0 \pm 9.6 \%$ \\
\hline $\begin{array}{c}\text { Au-modified/Swipe } \\
6 \text { classes }\end{array}$ & $80.0 \%$ & $80.0 \%$ & $95.0 \%$ & $85.0 \pm 12.9 \%$ & $85.0 \pm 12.9 \%$ & $85.0 \pm 12.9 \%$ \\
\hline $\begin{array}{c}\text { Au-modified/Swipe } \\
3 \text { classes }\end{array}$ & $100 \%$ & $100 \%$ & $100 \%$ & $95.0 \pm 10.5 \%$ & $95.0 \pm 10.5 \%$ & $95.0 \pm 10.5 \%$ \\
\hline
\end{tabular}




\section{Figures}
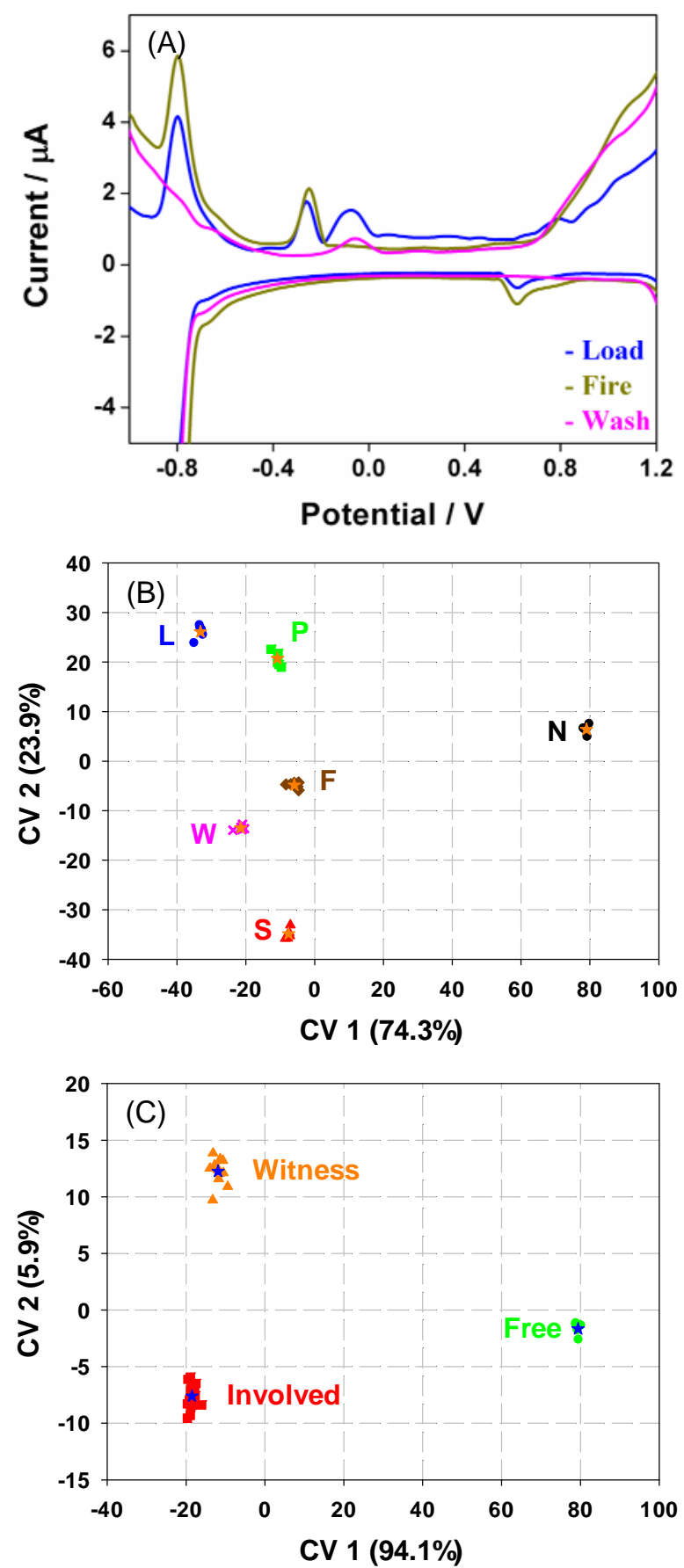

Figure 1. (A) Example of the different cyclic square-wave stripping voltammetric signals obtained with "swiping" samples at a bare SPCE electrode. Score plot of the variables obtained after CVA analysis of the GSR samples according to (B) exposure level or (C) 3-class response mode. Samples in (B) correspond to: $N$ - no contact, $S$ - Secondary exposure, $P$ - presence at discharge, $L$ - load, $F$ - fire and $W$ - wash. Samples in (C) correspond to Free $(N)$, Witness $(S \& P)$ and Involved $(L, F \& W)$. See Section 2.3 for the electrochemical conditions. 

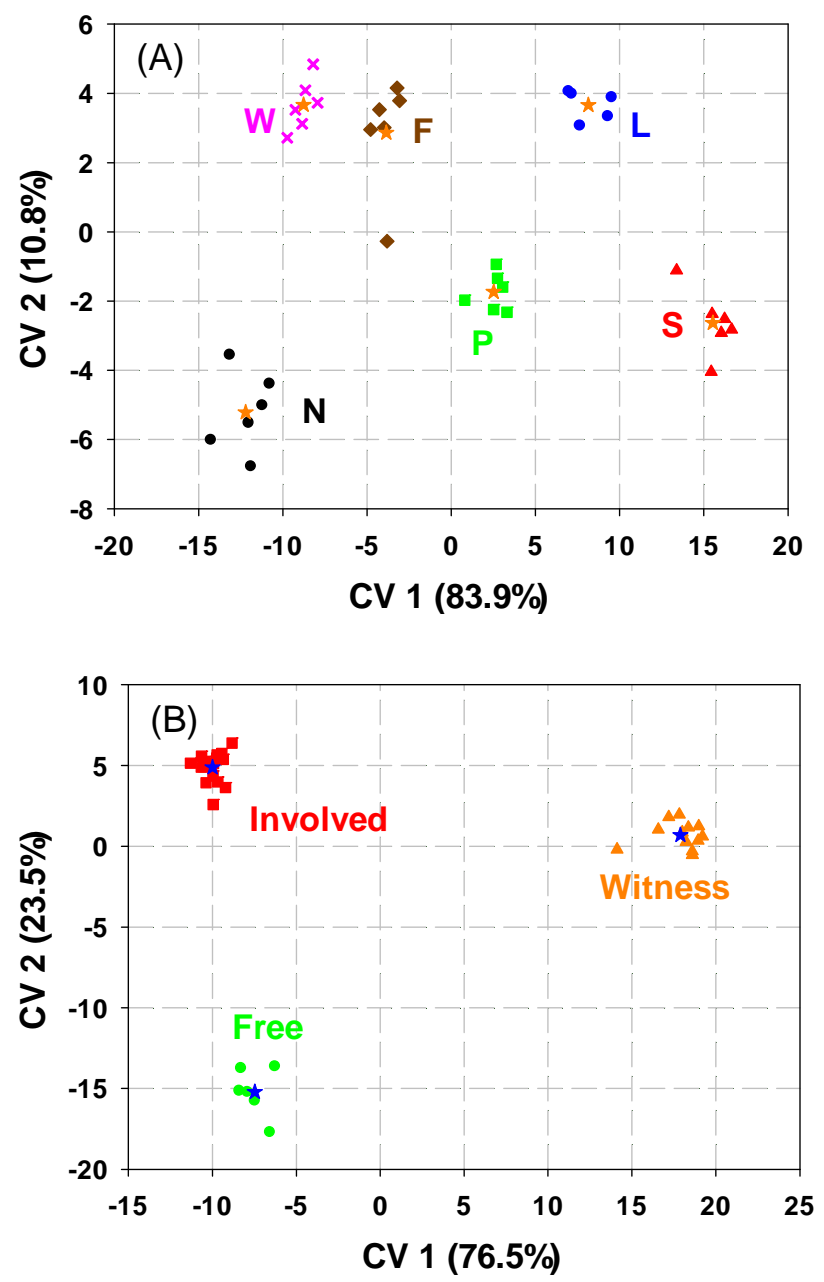

Figure 2. Score plot of the variables obtained after CVA analysis of the GSR samples according to (A) exposure level or (B) 3-class response mode. Samples correspond to the same controls outlined in Figure 1. 

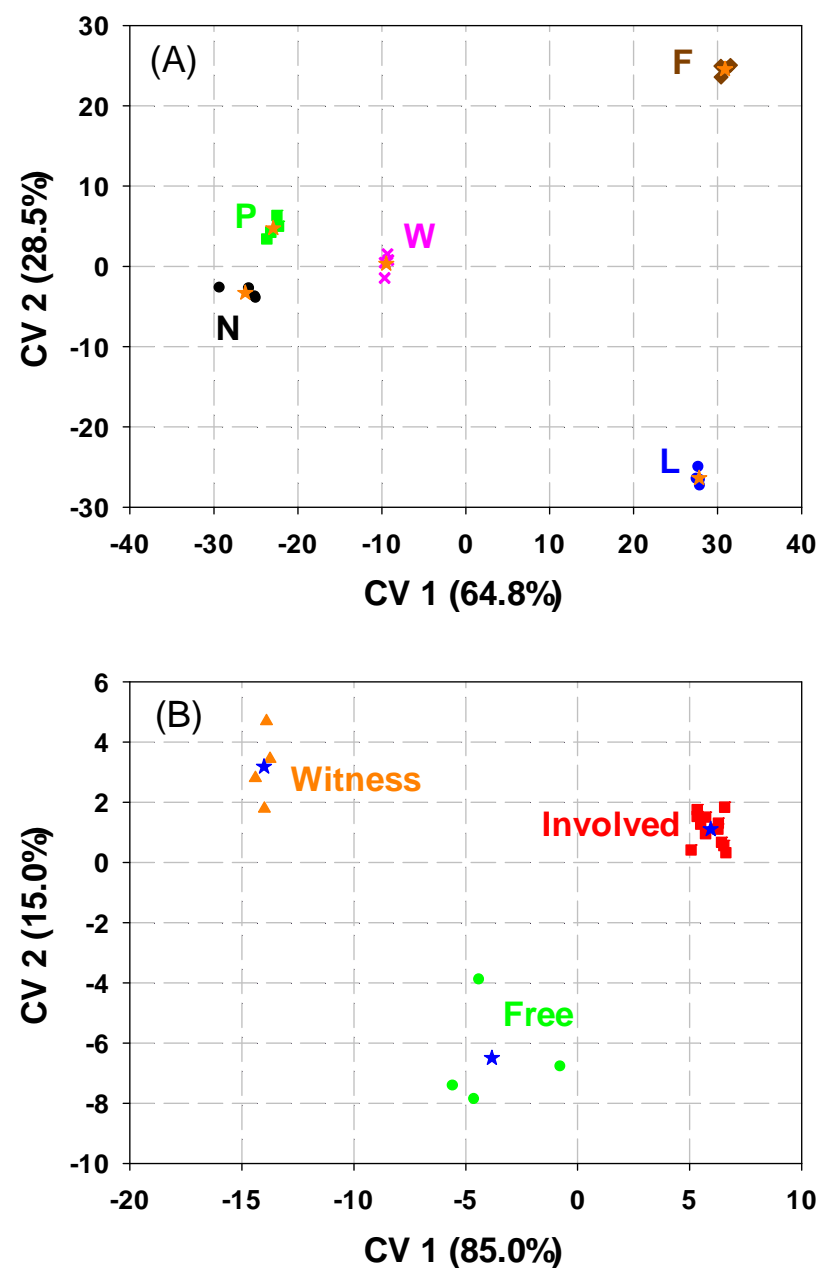

Figure 3. Score plot of the variables obtained after CVA analysis of the GSR samples according to (A) exposure level or (B) 3-class response mode. Samples in (A) correspond to: $N$ - no contact, $P$ - presence at discharge, $L$ - load, $F$ - fire and $W$ - wash. Samples in (B) correspond to Free $(N)$, Witness $(P)$ and Involved $(L, F \& W)$. 


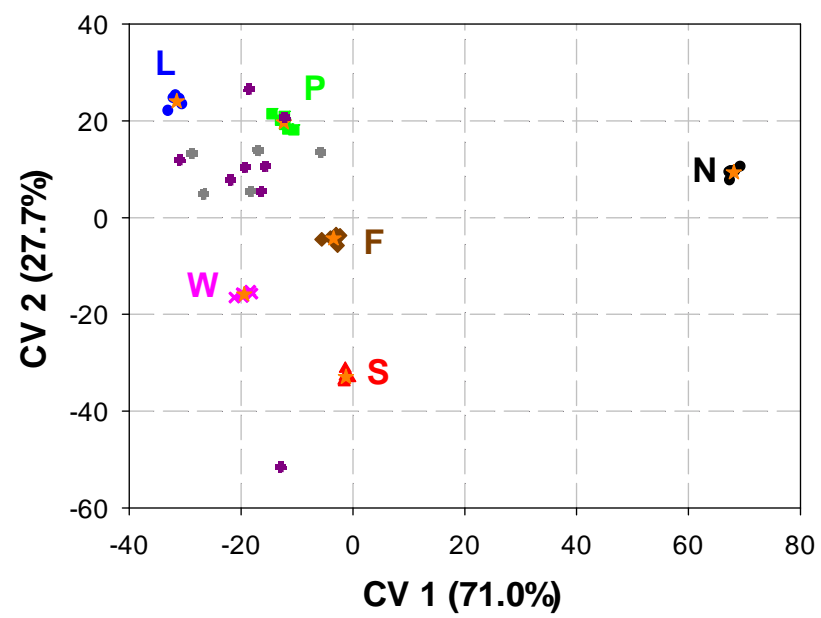

Figure 4: Score plot of the variables obtained after CVA analysis of the GSR samples according to exposure level outlined in Figure 1. Data points in purple and grey represent samples taken from machineshop technicians and smokers, respectively. 